\title{
THE VARIATION OF RESIDUAL INDUCTION AND COERCIVE FORCE WITH MAGNETIZING FORCE
}

\author{
By R. L. Sanford and W. L. Cheney
}

An analytical expression representing the relationship between magnetic quantities for ferromagnetic substances has been sought by many investigators. The law of Fröhlich, however, which was later modified by Kennelly" is the "only rational law of magnetic relation which has not been disproven." 2 This law states that the "permeability is proportional to the magnetizability." That is,

$$
\mu=a(S-B)
$$

where $\mu$ is the permeability, $S$ is the saturation value of flux density, and $B$ is the flux density.

If in equation (I) we substitute the relation

$$
\mu=\frac{B}{H},
$$

we have, upon rearranging,

$$
B=\frac{H}{a+\sigma H},
$$

where $\sigma=\frac{I}{S}=$ saturation coefficient, or reciprocal of the saturation density $S$, and $a=\frac{\mathrm{I}}{a S}=\frac{\sigma}{a}$. Kennelly's modification consisted of the introduction of the idea of reluctivity $(\rho)$, which is the reciprocal of permeability. By substituting the relation

$$
\rho=\frac{\mathrm{I}}{\mu}=\frac{H}{B}
$$

in equation (3) we can derive the equation

$$
\rho=a+\sigma H .
$$

\footnotetext{
1 Keninelly, Trans. A. I. E. E., 8, p. 485; I891. $181117^{\circ}-20$

2 Steinmetz, G. E., Rev., 20, p. 135; 1917. 
Since $B$ never reaches a saturation value or maximum, this equation was changed to the form

$$
\rho_{0}=a+\sigma H
$$

where $\rho_{0}$ is the metallic reluctivity $\left(\rho_{0}=\frac{H}{B-H}\right)$. This is the final expression of Kennelly's law and has been found to hold except for points near the origin for pure homogeneous materials.

If the values of residual induction $\left(B_{\mathrm{r}}\right)$ and coercive force $\left(H_{\mathrm{c}}\right)$ are plotted against maximum magnetizing force $\left(H_{\mathrm{m}}\right)$ as the independent variable, the resulting curves have the same characteristics as the curve of $B-H$ plotted against $H$. In view of this fact, it appears that there should hold for these quantities a relationship similar to that expressed by the reluctivity law of Kennelly.

In order to ascertain whether such a relationship does hold, hysteresis measurements have been made on a variety of materials ranging from Norway iron to an exceptionally hard steel, known as K S magnet steel, which was prepared by Prof. Honda. The magnetizing forces used ranged from small values up to about 2500 gausses, which in most cases was sufficient to produce magnetic saturation. The apparatus used in these experiments has already been fully described. ${ }^{3}$

Complete data for four typical specimens are given in Tables I to 4 and are plotted in the corresponding Figs. I to 4 . The data include observed values of $H_{\mathrm{m}}, B_{\mathrm{m}}, B_{\mathrm{r}}$, and $H_{\mathrm{c}}$ and calculated values of $\rho_{\mathrm{o}}, H_{\mathrm{m}} / B_{\mathrm{r}}$, and $H_{\mathrm{m}} / H_{\mathrm{c}}$. The actual observations are indicated in the graphs, and it is evident that the straight-line relationship holds within the limits of experimental error. Figs. 5 and 6 show curves for a number of other materials. In the case of Norway iron, the failure of the points for the $H_{\mathrm{m}} / H_{\mathrm{o}}$ curve to fall on a straight line, and the consequent uncertainty in the calculation of the maximum value of $H_{\mathrm{c}}$, are in all probability due to the fact that the sensitivity of the apparatus does not permit of determinations of very small values of $H_{\mathrm{c}}$ with as great an accuracy as can be obtained in other cases. 


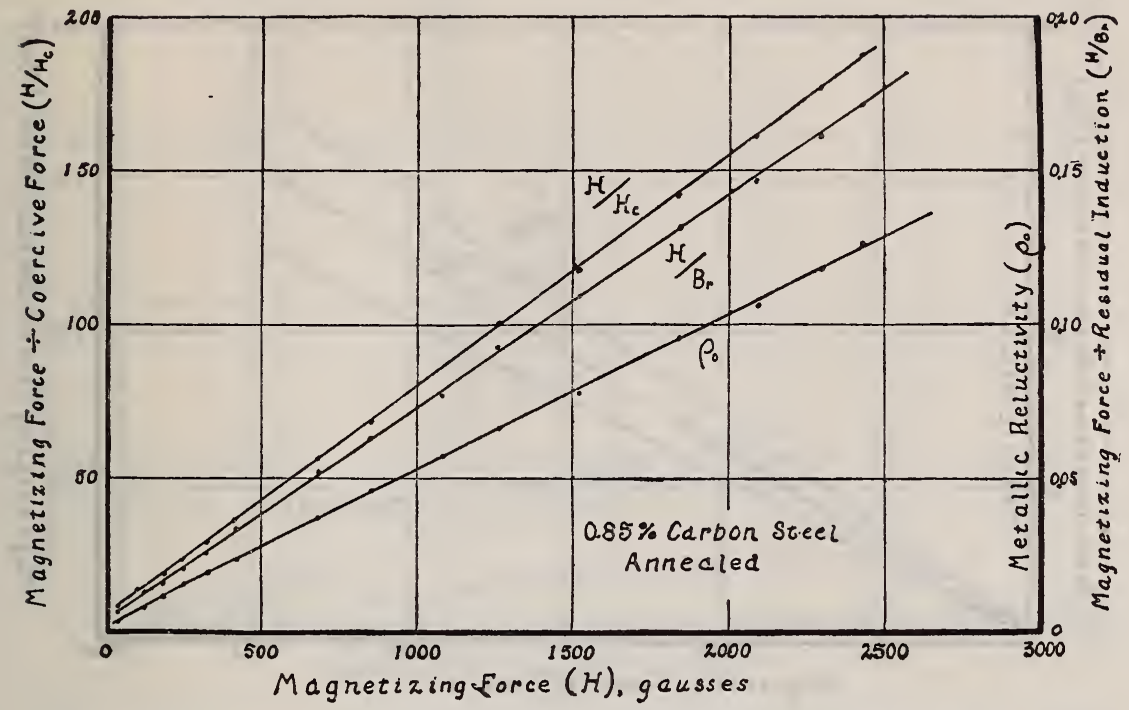

FIG. I.-Illustrating the magnetic properties of annealed 0.85 per cent carbon steel

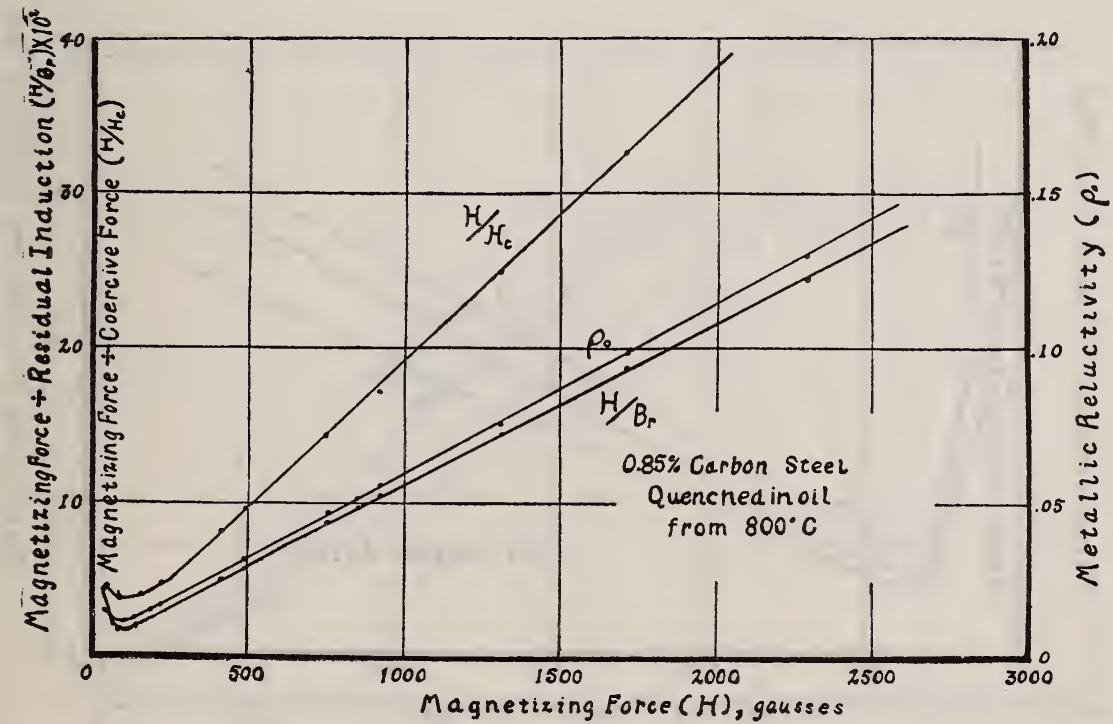

FIG. 2.-Illustrating the magnetic properties of 0.85 per cent carbon steel, quenched in oil from $800^{\circ} \mathrm{C}$ 


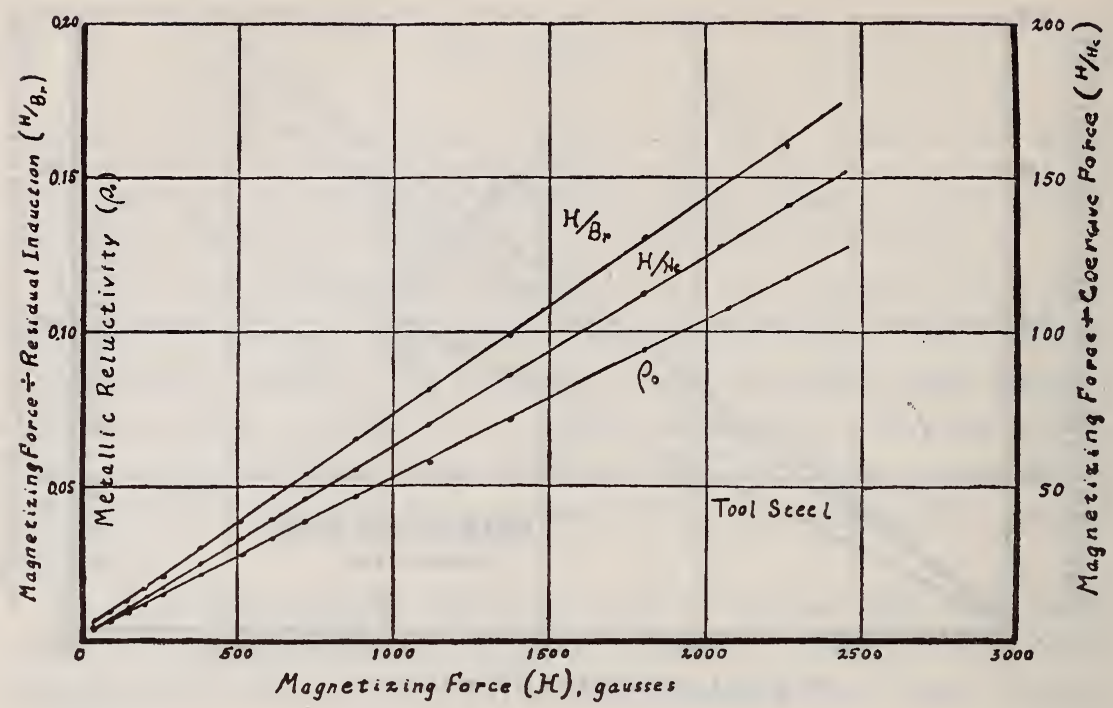

FIG. 3.-Illustrating the magnetic properties of tool steel, as received

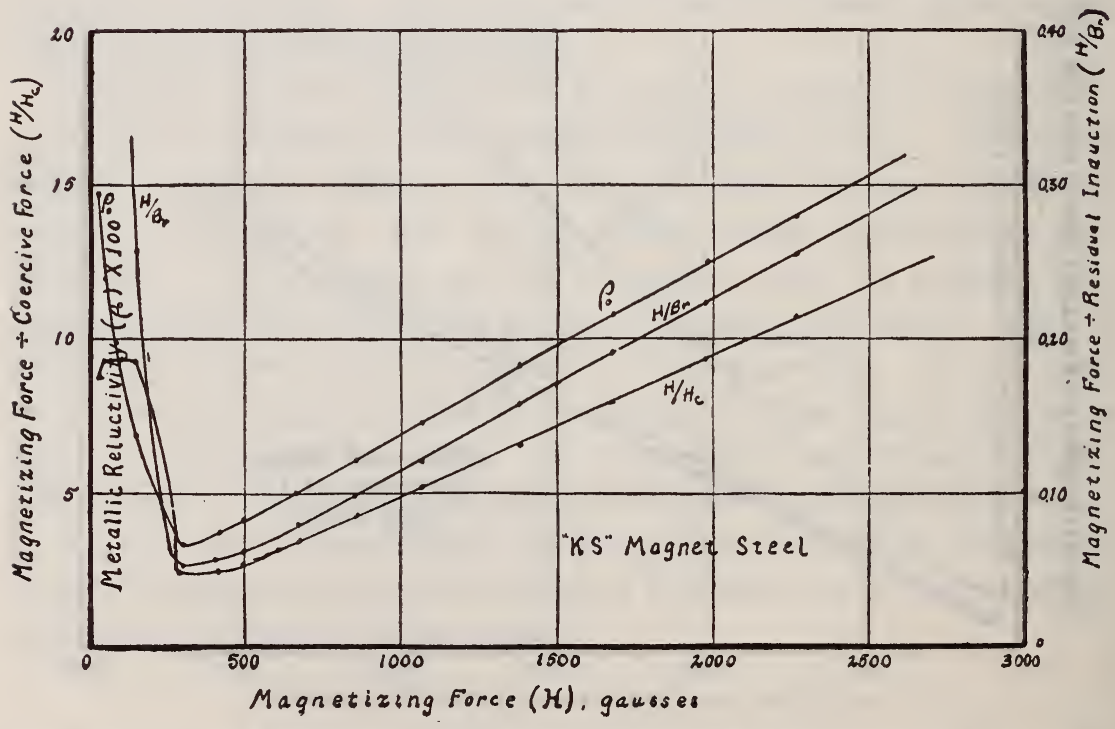

FrG. 4.-Illustrating the magnetic properties of hardened $K S$ magnet steel 


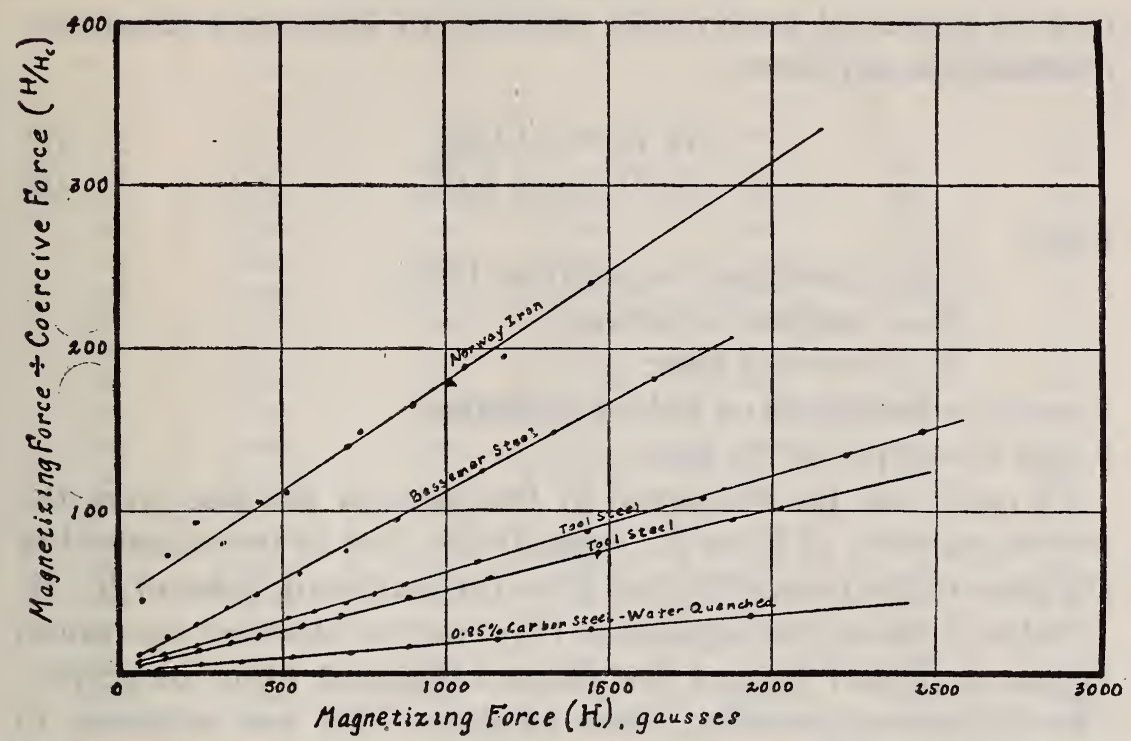

FIG. 5.-Showing the ratio $H_{m} / H_{c}$ plotted against the magnetizing force, for a number of materials

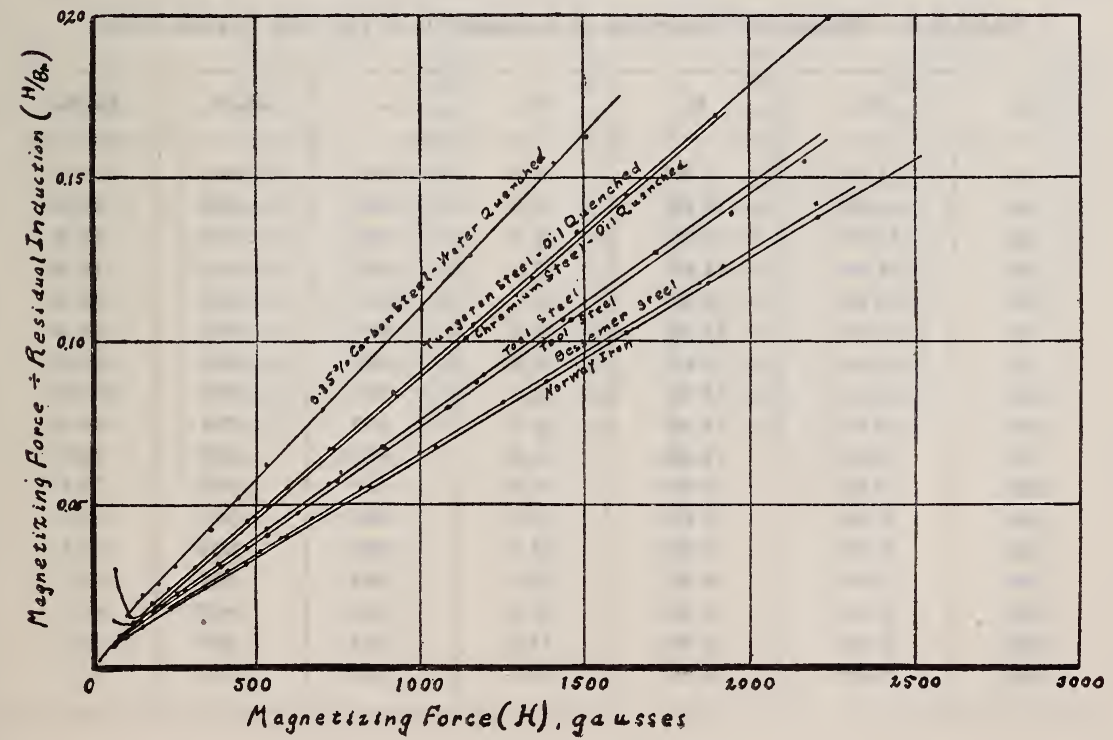

Fig. 6.-Showing the ratio $H_{m} / B_{r}$ plotted against the magnetizing force, for a number of materials 
This linear relationship, which the experiments have confirmed, may be expressed analytically similarly to Kennelly's reluctivity relationship, as follows:

$$
\begin{aligned}
& H_{\mathrm{m}} / B_{\mathrm{r}}=a_{1}+b_{1} H_{\mathrm{m}} \\
& H_{\mathrm{m}} / H_{\mathrm{c}}=a_{2}+b_{2} H_{\mathrm{m}}
\end{aligned}
$$

where

$H_{\mathrm{m}}=$ maximum magnetizing force,

$B_{\mathrm{r}}=$ residual induction,

$H_{\mathrm{c}}=$ coercive force,

$a_{1}$ and $a_{2}=$ intercepts on axis of ordinates,

$b_{1}$ and $b_{2}=$ slopes of the lines.

In each case the reciprocal of the slope of the line gives the maximum value of $B_{\mathrm{r}}$ or $H_{\mathrm{c}}$, respectively, just as the reciprocal of the slope of the reluctivity line gives the maximum value of $B-H$.

Table 5 shows the agreement between the observed maximum values of $B_{\mathrm{r}}$ and $H_{\mathrm{c}}$ and the values calculated from the graphs. The highest magnetizing force employed was not sufficient to saturate the K S magnet steel so that the calculated value of the maximum $H_{\mathrm{c}}$ is higher than the highest observed value. In all other cases, the calculated values agree well within the limits of the probable experimental error.

\begin{tabular}{|c|c|c|c|c|c|c|}
\hline $\mathbf{H}_{\mathbf{m}}$ & $\mathbf{B}_{\mathbf{m}}$ & $\mathrm{B}_{z}$ & $\mathbf{H}_{0}$ & $\rho_{0}$ & $\mathbf{H}_{m} / \mathbf{B}_{z}$ & $\mathbf{H}_{m} / \mathbf{H}_{0}$ \\
\hline 32 & 12080 & 7500 & 5.0 & 0.0027 & 0.0043 & 6. 40 \\
\hline 92 & 15020 & 10980 & 6.5 & .0062 & .0084 & 14. 15 \\
\hline 120 & 15570 & 11650 & 9.0 & .0078 & .0130 & 13. 35 \\
\hline 183 & 16280 & 12100 & 10.0 & .0114 & .0151 & 18.30 \\
\hline 246 & 16990 & 12550 & 10.5 & .0148 & .0196 & 23.40 \\
\hline 325 & 17690 & 13000 & 11.0 & .0187 & .0250 & .29 .55 \\
\hline 418 & 18140 & 12620 & 11.5 & .0236 & .0332 & 36. 34 \\
\hline 500 & 18380 & 13100 & 12.0 & .0280 & .0382 & 41.65 \\
\hline 680 & 18970 & 13240 & 12.0 & .0370 & .0514 & 56.60 \\
\hline 850 & 19380 & 13480 & 12.5 & .0459 & .0630 & 68.0 \\
\hline 1080 & 19820 & 13600 & 14.0 & .0577 & .0794 & 77.0 \\
\hline 1260 & 20350 & 13650 & 12.5 & .0660 & .0924 & 100.7 \\
\hline 1520 & 20890 & 14050 & 13.0 & .0785 & .1082 & 116.8 \\
\hline 1840 & 21240 & 14000 & 13.0 & .0948 & .1314 & 141.5 \\
\hline 2090 & 21930 & 14310 & 13.0 & .1053 & .1460 & 160.8 \\
\hline 2300 & 21940 & 14300 & 13.0 & .1172 & .1610 & 177.0 \\
\hline 2430 & 21860 & 14200 & 13.0 & .1250 & .1710 & 187.0 \\
\hline
\end{tabular}

TABLE 1.-Magnetic Properties of Annealed 0.85 per cent Carbon Steel 
TABLE 2.-Magnetic Properties of 0.85 per cent Carbon Steel, Quenched in Oil from $800^{\circ} \mathrm{C}$

\begin{tabular}{|c|c|c|c|c|c|c|}
\hline $\mathbf{H}_{m}$ & $\mathbf{B}_{m}$ & $\mathbf{B}_{\mathbf{r}}$ & $\mathbf{H}_{0}$ & $p_{0}$ & $\mathbf{H}_{m} / \mathbf{B}_{\boldsymbol{r}}$ & $\mathbf{E}_{\mathbf{m}} / \mathbf{H}_{0}$ \\
\hline 42 &.$\quad 2720$ & 950 & 9 & 0.0157 & 0.044 & 4.67 \\
\hline 86 & 8050 & 4540 & 22 & .0108 & .0190 & 3.91 \\
\hline 137 & 11210 & 6900 & 34 & .0124 & .0199 & 4.03 \\
\hline 190 & 12680 & 7600 & 44.5 & .0152 & .0250 & 4.27 \\
\hline 220 & 13160 & 7700 & 46.0 & .0170 & .0286 & 4.78 \\
\hline 304 & 14360 & 8150 & 49.5 & .0216 & .0373 & 6.14 \\
\hline 410 & 15240 & 8300 & 50.0 & .0276 & .0494 & 8.20 \\
\hline 485 & 15690 & 8440 & 50.5 & .0318 & .0575 & 9.60 \\
\hline 570 & 16290 & 8550 & 52.0 & .0363 & .0667 & 10.95 \\
\hline 670 & 16580 & 8600 & 52.0 & .0421 & .0780 & 12.88 \\
\hline 748 & 16660 & 8600 & 52.0 & .0470 & .0870 & 14. 38 \\
\hline 842 & 17220 & 8750 & 52.0 & .0514 & .0963 & 16.20 \\
\hline 920 & 17420 & 8800 & 53.0 & .0558 & .1045 & 17.35 \\
\hline 1050 & 18040 & 8900 & 52.5 & .0618 & .1180 & 20.00 \\
\hline 1310 & 18570 & 9000 & 52.5 & .0760 & .1455 & 25.00 \\
\hline 1500 & 18750 & 9100 & 53.0 & .0870 & .1650 & 28. 30 \\
\hline 1710 & 19000 & 9120 & 52.5 & .0990 & .1875 & 32.60 \\
\hline 2000 & 19540 & 9300 & 52.5 & .1140 & .2150 & 38.10 \\
\hline 2290 & 19930 & 9380 & 52.0 & .1300 & .2440 & 44.00 \\
\hline 2420 & 19970 & 9300 & 52.5 & .1378 & .2600 & 46.10 \\
\hline
\end{tabular}

TABLE 3.-Magnetic Properties of Tool Steel, as Received

\begin{tabular}{|c|c|c|c|c|c|c|}
\hline $\mathbf{H}_{m}$ & $\mathbf{B}_{\text {ma }}$ & $\mathbf{B}_{\mathbf{r}}$ & $\mathbf{H}_{0}$ & $\rho_{0}$ & $\mathbf{H}_{\mathbf{m}} / \mathbf{B}_{\boldsymbol{r}}$ & $\mathbf{H}_{\mathbf{m}} / \mathbf{H}_{0}$ \\
\hline 34 & 10680 & 6500 & 8.0 & 0.0032 & 0.0052 & 4. 25 \\
\hline 90 & 14740 & 9840 & 10.0 & .0061 & .0092 & 9.00 \\
\hline 144 & 16010 & 11100 & 13.5 & .0091 & .0130 & 10.65 \\
\hline 202 & 17170 & 12100 & 14.0 & .0119 & .0167 & 14.43 \\
\hline 260 & 17340 & 12300 & 14.5 & .0152 & .0211 & 17.90 \\
\hline 380 & 18160 & 12600 & 15.0 & .0214 & .0302 & 25.35 \\
\hline 510 & 18680 & 13000 & 15.5 & .0280 & .0392 & 32.90 \\
\hline 610 & 19210 & 13200 & 15.5 & .0328 & .0462 & 39.40 \\
\hline 710 & 19200 & 13090 & 15.5 & .0384 & .0543 & 45.8 \\
\hline 870 & 19750 & 13300 & 16.0 & .0461 & .0654 & 54.4 \\
\hline 1110 & 20300 & 13600 & 16.0 & .0579 & .0816 & 69.4 \\
\hline 1370 & 20630 & 13800 & 16.0 & .0711 & .0994 & 85.7 \\
\hline 1800 & 21060 & 13800 & 16.0 & .0935 & .1310 & 112.5 \\
\hline 2260 & 21770 & $1+100$ & 16.0 & .1175 & .1600 & 141.0 \\
\hline
\end{tabular}


TABLE 4.-Magnetic Properties of Hardened K S Magnet Steel

\begin{tabular}{|c|c|c|c|c|c|c|}
\hline $\mathbf{H}_{\mathbf{m}}$ & $\mathbf{B}_{m}$ & $\mathrm{~B}_{\mathbf{z}}$ & $\mathbf{H}_{0}$ & $\rho_{0}$ & $\mathrm{H}_{\mathrm{m}} / \mathrm{B}_{\mathrm{z}}$ & $\mathbf{H}_{m} / \mathbf{H}_{0}$ \\
\hline 26 & 210 & 0 & & 0.1470 & & \\
\hline 48 & 450 & 80 & 5.5 & .1195 & 0.6000 & 8.73 \\
\hline 84 & ........ & & 9 & ........ & •........ & 9. 34 \\
\hline 100 & 1140 & 200 & ...... & .0962 & .5000 & .......... \\
\hline 149 & 2280 & 580 & 16 & .0694 & .2569 & 9. 32 \\
\hline 224 & 4660 & 2100 & 48 & .0505 & .1074 & 4.67 \\
\hline 296 & 9080 & 5610 & 116 & .0337 & .0528 & 2. 55 \\
\hline 410 & 11340 & 7200 & 160 & .0375 & .0570 & 2.56 \\
\hline 490 & 12290 & 7800 & 178 & .0415 & .0628 & 2. 75 \\
\hline 665 & 13780 & 8360 & 194 & .0506 & .0795 & 3.43 \\
\hline 850 & 14710 & 8650 & 201 & .0613 & .0982 & 4.23 \\
\hline 1060 & 15530 & 8800 & 205 & .0733 & .1205 & 5.17 \\
\hline 1380 & 16510 & 8780 & 210 & .0912 & .1572 & 6.57 \\
\hline 1675 & 17190 & 8850 & 210 & .1080 & .1893 & 7.97 \\
\hline 1975 & 17750 & 8840 & 211 & .1250 & .2240 & 9.36 \\
\hline 2260 & 18370 & 8900 & 212 & 1400 & .2540 & 10.70 \\
\hline 2500 & 18720 & 8900 & 215 & .1540 & .2820 & 11.62 \\
\hline
\end{tabular}

TABLE 5.-Showing the Agreement between Calculated and Observed Values of Maximum Residual Induction and Maximum Coercive Force for a Number of Specimens

\begin{tabular}{|c|c|c|c|c|}
\hline \multirow{2}{*}{ Material } & \multicolumn{2}{|c|}{ Maximum $\mathbf{B}_{\boldsymbol{r}}$} & \multicolumn{2}{|c|}{ Maximum $\mathbf{H}_{\mathrm{c}}$} \\
\hline & Calculated & Observed & Calculated & Observed \\
\hline Norway iron................. & 16400 & 15900 & 7.4 & 6.0 \\
\hline Bessemer steel......................... & 16000 & 15900 & 9.4 & 9.0 \\
\hline 0.85 per cent carbon steel (annealed)....... & 14500 & 14310 & 13.5 & 13.0 \\
\hline 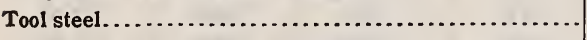 & 14200 & 14100 & 16.2 & 16.0 \\
\hline 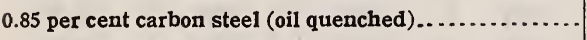 & 9550 & 9380 & 52.7 & 53.0 \\
\hline Chromium steel (oil quenched)................ & 11300 & 11200 & 53.8 & 53.5 \\
\hline 0.85 per cent carbon steel (water quenched).............. & 9750 & 9800 & 57.0 & 56.5 \\
\hline Tungsten steel (oil quenched) $. . . \ldots \ldots \ldots \ldots \ldots \ldots \ldots . . . . . . .$. & 11300 & 11200 & 69.0 & 68.0 \\
\hline K S magnet steel....................................... & 9000 & 8900 & 220 & $a 215$. \\
\hline
\end{tabular}

$a$ Saturation not reached.

WASHINGTON, March 20, I920. 


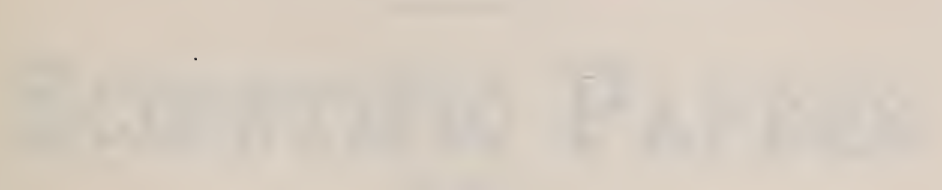

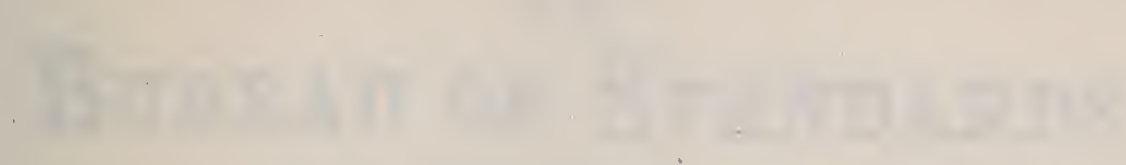





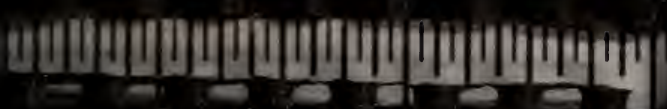

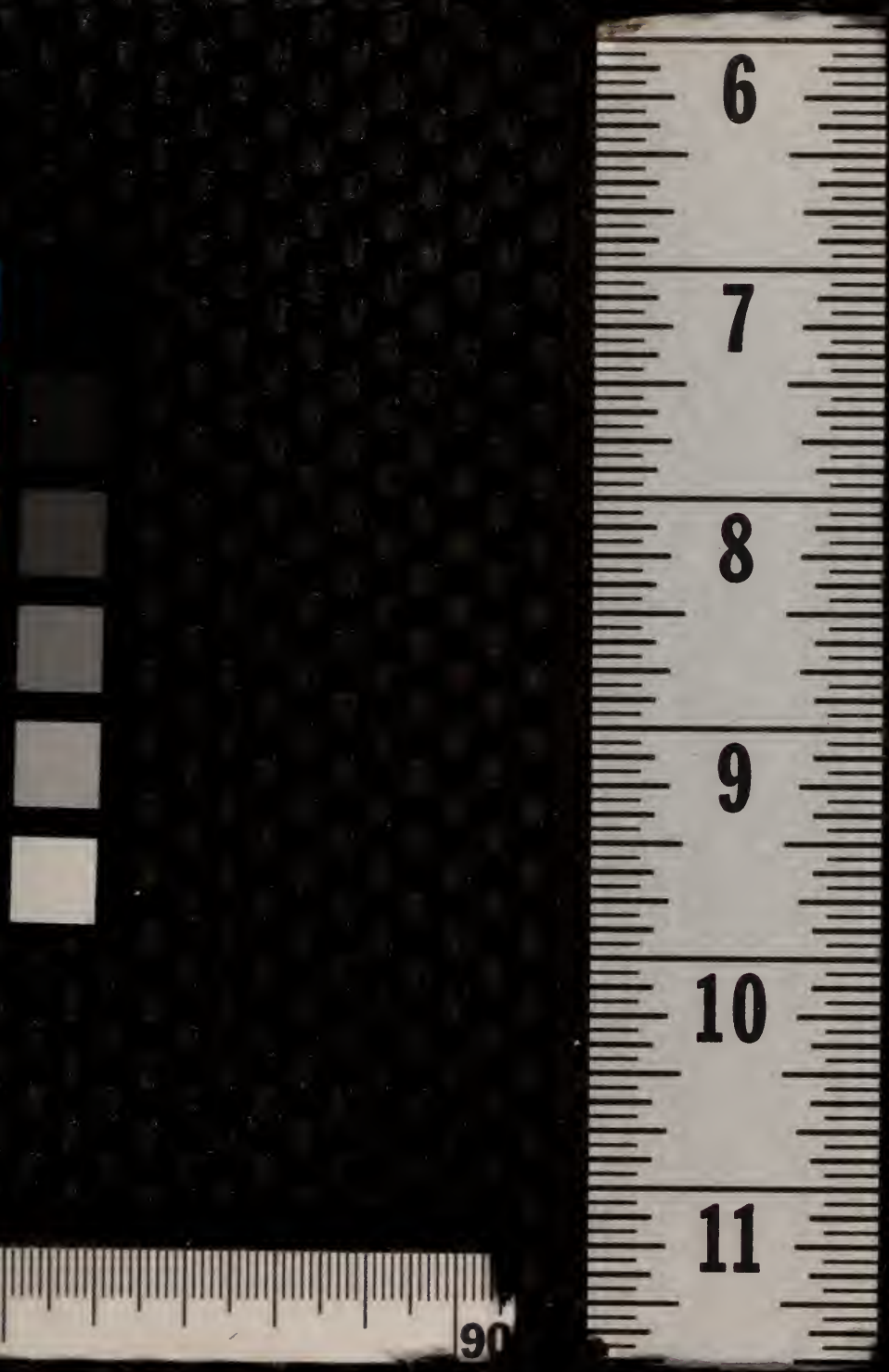

